\title{
Electrolytic lesions of the medial prefrontal cortex do not interfere with long-term memory of extinction of conditioned fear
}

\author{
René Garcia, ${ }^{1,4}$ Chun-hui Chang, ${ }^{2}$ and Stephen Maren ${ }^{2,3}$ \\ ${ }^{1}$ Laboratory of Neurobiology and Psychopathology, University of Nice-Sophia Antipolis, 06108 Nice, France; ${ }^{2}$ Department \\ of Psychology and ${ }^{3}$ Neuroscience Program, University of Michigan, Ann Arbor, Michigan 48109, USA
}

\begin{abstract}
Lesion studies indicate that rats without the medial prefrontal cortex (mPFC) have difficulty recalling fear extinction acquired the previous day. Several electrophysiological studies have also supported this observation by demonstrating that extinction-related increases in neuronal activity in the mPFC participate in expression of fear extinction. However, a more recent study has shown that fear extinction can be recalled, in certain circumstances, without mPFC potentiation, suggesting contribution of other circuits. Here, we examined this possibility in rats that were subjected to auditory fear conditioning, extinction training, and extinction retention test $7 \mathrm{~d}$ later. Electrolytic lesions were made in the mPFC, the motor cortex (MO), the dorsal septum (SEP), or the mediodorsal thalamus (MD), because of their potential participation in conditioned fear inhibition; combined lesions including the mPFC with the $\mathrm{MO}$, SEP, or MD were also made. The lesions were made either $1 \mathrm{wk}$ before conditioning or $1 \mathrm{~d}$ after extinction training. All rats normally extinguished their conditioned freezing behavior during extinction training and did not display any return of this behavior during the retention test. These data reveal that the mPFC is not required for the acquisition, the expression, or the retrieval of extinction memories but do not exclude the possibility that the mPFC normally participates in these processes.
\end{abstract}

Freezing behavior evoked by a tone paired with footshock decreases in strength when the tone is repeatedly presented without the footshock. This phenomenon, known as extinction (Pavlov 1927), recruits both short- and long-term memory processes. Several studies have indicated that the medial prefrontal cortex (mPFC) may be a key structure in processing long-term memory of extinction of conditioned fear by inhibiting post-extinction expression of fear memory, which is known to persist after extinction (e.g., Garcia 2002). For example, it has been shown that lesions of the mPFC leave intact (Gewirtz et al. 1997; Quirk et al. 2000; Lebron et al. 2004) or delay (Morgan et al. 1993; Morgan and LeDoux 1995) short-term processes of extinction memory but produce deficits in the long-term retention of extinguished fear (Quirk et al. 2000; Lebron et al. 2004).

In electrophysiological studies, it has been shown that extinction of conditioned fear is accompanied by increased neuronal activity in the mPFC that is not required for extinction learning per se (Herry et al. 1999), but contributes to inhibition of spontaneous recovery of the extinguished fear (Herry and Garcia 2002; Milad and Quirk 2002). Indeed, both prefrontal long-term potentiation (LTP) and long-term depression (LTD), induced by train stimulation of the mediodorsal thalamus (MD), were found to have no effect on the rate of extinction of conditioned fear (short-term processes of extinction memory) but were associated with inhibition (LTP) and facilitation (LTD) of spontaneous recovery (Herry and Garcia 2002). However, in a more recent study analyzing synaptic plasticity changes in the projection from the hippocampus (HPC) to the mPFC, contradictory results have been found (M. Farinelli, O. Deschaux, S. Hugues, and R. Garcia, in prep.). First, this study reveals that the HPC-mPFC pathway also displays LTP-like changes following extinction training. Sec-

\section{${ }^{4}$ Corresponding author.}

E-mail rgarcia@unice.fr; fax 33-492-07-61-62.

Article published online ahead of print. Article and publication date are at http://www.learnmem.org/cgi/doi/10.1101//m.60406. ond, the same study also indicates that suppression of extinctionrelated HPC-mPFC LTP by HPC low-frequency stimulation, applied immediately after extinction training, is associated with facilitation of spontaneous recovery of conditioned freezing. However, in contrast to previous findings, Farinelli and colleagues' study (M. Farinelli, O. Deschaux, S. Hugues, and R. Garcia, in prep.) shows that HPC tetanus, applied $72 \mathrm{~h}$ after HPC low-frequency stimulation, can inhibit the return of the conditioned fear behavior without restoring HPC-mPFC LTP, probably by inducing synaptic plasticity changes in other HPC output targets. This suggests that in certain circumstances, the integrity of these HPC output targets, but not of the MPFC, may be required for the expression of extinction memory.

To address this issue, we first examined in rats whether preconditioning or post-extinction lesions of the mPFC and other hippocampal output targets that have been implicated in conditioned fear inhibition (the motor cortex [MO] and MD [Barrett et al. 2003] and the dorsal septum [SEP] [Thomas et al. 1991]) would impair expression of memory of fear extinction. We also analyzed whether pre-conditioning or post-extinction lesions of the mPFC combined with lesions of the MO, the SEP, or the MD would have greater effects on long-term extinction memory. Animals were fear conditioned in one context (five tone-footshock pairings), placed in a different context the following day for extinction (45 tone-alone presentations), and tested for extinction retention $7 \mathrm{~d}$ after extinction training in the extinction context (five tone-alone presentations).

\section{Results}

\section{Histology}

Histological assessment of damage to the targeted brain regions is presented in Figure 1. Prefrontal lesions were localized predominantly to the prelimbic and infralimbic areas (mPFC lesions) and the medial and lateral pre-central areas (MO lesions) of the PFC. MD lesions were localized in the medial part of the MD. Septal lesions (SEP) were localized in the dorsal lateral septum and in- 


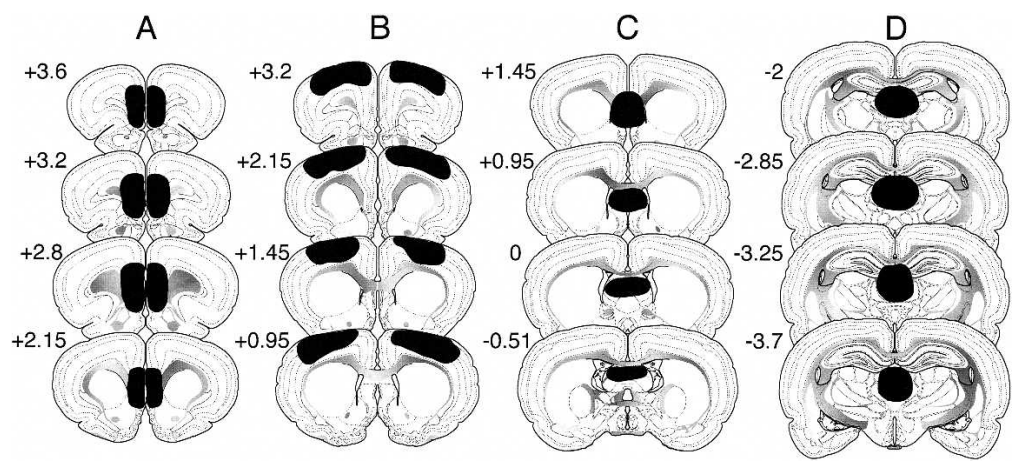

Figure 1. Histology. Schematic representation of brain lesions mapped onto coronal rat brain sections showing the extent of the electrolytic lesions (plotted in black) in the prefrontal infralimbic and prelimbic areas $(A)$, motor cortex $(B)$, septal area $(C)$, and mediodorsal thalamus $(D)$.

cluded also the most-dorsal part of the medial septum. Animals with misplaced lesions or without bilateral lesions were excluded from the statistical analyses.

\section{Single-site lesions do not impair expression of fear extinction}

Un-operated (UN-OP) and sham-operated (SH) rats and those with lesions (mPFC, MO, SEP, and MD) exhibited high levels of freezing to the CS early in the extinction training session and extinguished this response over the course of the session (Fig. 2A). Freezing was low during the entire session in rats that did not receive CS presentations (NO-EXT). Statistical analyses of these data revealed a significant group $\times$ extinction block interaction $\left(F_{(54,747)}=2.7 ; P<0.0001\right)$. There was no effect of lesion in the analysis indicating that short-term extinction was normal in all of the groups.

During the retention test, only sham-operated rats that did not receive tone presentations during the extinction training (NO-EXT) exhibited high levels of freezing. Freezing levels in the remaining groups that were subjected to extinction training $7 \mathrm{~d}$ earlier remained low (Fig. 2B). Statistical analyses on these data confirmed that all animals submitted to extinction training did not differ from each other during the retention test. However, each of these groups differed from the NO-EXT group during the retention test (all $P \mathrm{~s}<0.001$ ). These data indicate that, under our experimental conditions, neither pre-conditioning nor postextinction lesions of the MPFC, MO, SEP, or MD interfered with the consolidation of fear extinction.

\section{Combined lesions including the mPFC do not impair expression of fear extinction}

We hypothesized that the failure of mPFC lesions to impair the retention of extinction may have been due to compensation from the MO, the SEP, the MD, or other brain regions. We therefore combined mPFC lesions with lesions of the MO $(\mathrm{MO}+\mathrm{mPFC})$, the SEP (SEP + mPFC), or the MD (MD + mPFC). Rats in all of the lesion groups and $\mathrm{SH}$ and $\mathrm{UN}-\mathrm{OP}$ rats displayed high levels of freezing early in extinction training and extinguished this response with repeated presentations of the tone CS; NO-EXT rats exhibited low levels of freezing during the entire session (Fig. 3A). Statistical analyses of these data revealed a significant interaction between group and extinction block $\left(F_{(45,540)}=5.1 ; P<0.0001\right)$. The NO-EXT rats differed $(P \mathrm{~S}<0.05)$ from all of the other groups that did not differ from each other.

During the retention tests, only SH rats that did not receive tone presentations during extinction training (NO-EXT group) displayed high levels of freezing, while freezing levels were low in are believed to contribute to inhibition of conditioned fear (i.e., $\mathrm{mPFC}, \mathrm{MO}, \mathrm{SEP}$, or MD) or lesions of the MPFC combined with one of these areas (MO, SEP, or MD). Animals were tested for retention of fear extinction $7 \mathrm{~d}$ after extinction training. None of the lesions affected the expression of extinction during the retention test, indicating normal recall of fear extinction. Together with previous findings, these results suggest that prefrontal areas such as infralimbic and prelimbic areas and MO may be involved in, but are not necessary for either the acquisition or expression of short- and long-term extinction memory.

At present, it is not clear what conditions are necessary to observe an impairment in fear extinction with lesions in the mPFC. LeDoux and colleagues (Morgan et al. 1993; Morgan and

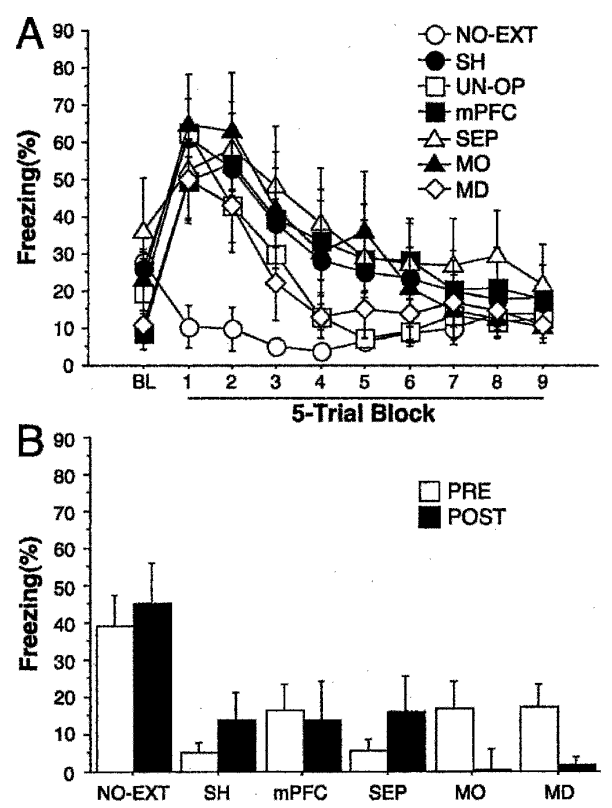

Figure 2. (A) Extinction training: Freezing behavior (mean \pm s.e.m.) during the first minute preceding the first CS-alone presentation (BL, baseline) and during CS-alone presentations (45 trials averaged in five trial blocks) in rats that received pre-training lesions of the medial prefrontal cortex (mPFC) $(n=8)$, motor cortex (MO) $(n=8)$, septal area (SEP) $(n=8)$, or mediodorsal thalamus (MD) $(n=6)$, and sham-operated $(\mathrm{SH})(n=11)$ and un-operated (UN-OP) $(n=33)$ rats. (B) Retention test: freezing behavior (mean \pm s.e.m.) during each CS-alone presentation (five trials) in subjects operated before conditioning (PRE) or after extinction (POST) (mPFC, $n=7$; MO, $n=6$; SEP, $n=6$; MD, $n=7$; SH, $n=7$ ). The NO-EXT group $(n=16)$ represents rats that were also operated before conditioning (PRE) $(n=12)$ or after extinction (POST) $(n=4)$ but did not receive $\mathrm{CS}$-alone presentations during extinction training. 

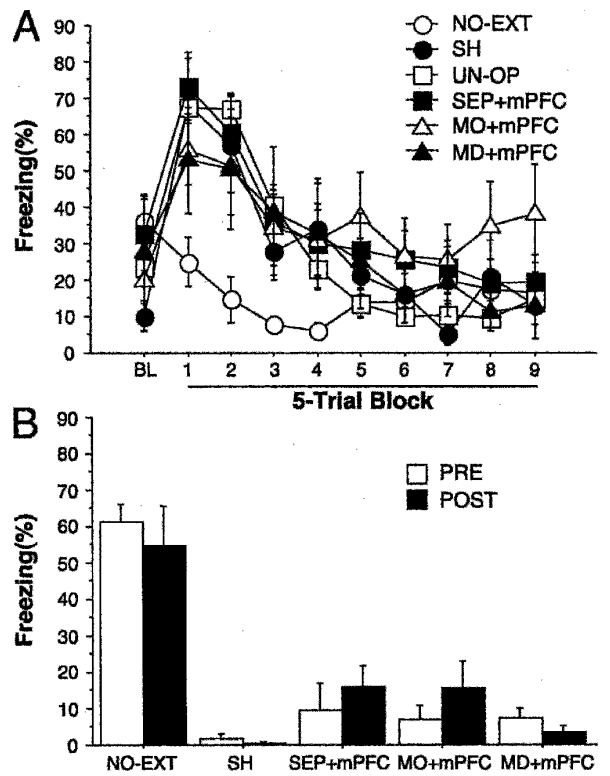

Figure 3. (A) Extinction training: Freezing behavior (mean \pm s.e.m.) during the first minute preceding the first CS-alone presentation ( $\mathrm{BL}$, baseline) and during CS-alone presentations (45 trials averaged in five trial blocks) in rats that received pre-training combined lesions of the medial prefrontal cortex and the motor cortex $(\mathrm{MO}+\mathrm{mPFC})(n=8)$, the septal area (SEP + MPFC) $(n=8)$, or the mediodorsal thalamus $(\mathrm{MD}+\mathrm{mPFC})(n=5)$, and sham-operated $(\mathrm{SH})(n=7)$ and un-operated (UN-OP) $(n=23)$ rats. (B) Retention test: Freezing behavior (mean \pm s.e.m.) during each CS-alone presentation (five trials) in subjects operated on before conditioning (PRE) or after extinction (POST) $(\mathrm{MO}+\mathrm{mPFC}, n=7 ; \mathrm{SEP}+\mathrm{mPFC}, n=6 ; \mathrm{MD}+\mathrm{mPFC}, n=6 ; \mathrm{SH}, n=4)$. The NO-EXT group $(n=15)$ represents rats that were also operated on before conditioning (PRE; $n=7)$ or after extinction (POST) $(n=8)$ but did not receive $\mathrm{CS}$-alone presentations during extinction training.

LeDoux 1995) first demonstrated in rats that lesions of both infralimbic and prelimbic areas of the mPFC retard extinction of freezing behavior to a tone CS. However, two other studies later showed that rats without the infralimbic area (Gewirtz et al. 1997) and mice without the prelimbic area (Vouimba et al. 2000) can acquire extinction of conditioned fear. Others have found that the infralimbic area of the mPFC does not contribute to short-term extinction, but is involved in the retrieval of that extinction memory $24 \mathrm{~h}$ after extinction (Quirk et al. 2000; Lebron et al. 2004). The present data stand in contrast to these reports, insofar as we have observed normal extinction retention 1 wk after extinction in rats with damage to the mPFC. It is possible that pre-training lesions affected the strength of initial conditioned freezing behavior, which could have altered assessment of the effects of these lesions on extinction. However, there were no differences in conditioned freezing between the groups at the start of extinction (Fig. 2). Alternatively, the discrepancies between our data and previous findings (Quirk et al. 2000; Lebron et al. 2004) might be due to contextual variables (conditioning and extinction in the same context vs. conditioning and extinction in different contexts in the present study) or the delay between extinction training and testing $(1 \mathrm{~d}$ vs. $7 \mathrm{~d}$ in the present study). The mPFC may therefore participate in long-term extinction if present (as shown by other approaches) (see also Herry and Mons 2004; Hugues et al. 2004; Santini et al. 2004), but under certain experimental conditions other areas may assure the same function in the absence of the MPFC, as shown here.

Electrophysiological studies also show that extinction of conditioned fear can develop normally, whatever the level of neuronal activity in the mPFC. In particular, fear extinction can be associated with increases (Herry et al. 1999; Herry and Garcia 2002; Milad and Quirk 2002), decreases (Herry and Garcia 2002), or no change (Milad and Quirk 2002) in neuronal activity in the mPFC. In contrast, post-extinction prefrontal changes that develop after training are known to interact with long-term processes of fear extinction. Indeed, only animals with increased neuronal activity in the MPFC in the mouse prelimbic area (Herry and Garcia 2002) or the rat infralimbic area (Milad and Quirk 2002) were found to be capable of recalling extinction. Thus, these lesion and electrophysiological data contrast with the present study where both pre-conditioning and post-extinction lesions of the mPFC did not interfere with long-term fear extinction. However, our data are in accordance with more recent electrophysiological observations in which the synaptic potentiation in the mPFC and the expression of long-term extinction were dissociated (M. Farinelli, O. Deschaux, S. Hugues, and R. Garcia, in prep.). It is possible that when the mPFC is absent (e.g., the present study) or not fully functional (absence of potentiation of neuronal activity) (M. Farinelli, O. Deschaux, S. Hugues, and R. Garcia, in prep.), increased activity in other brain circuits may facilitate the expression of extinction memory.

Among other potential areas that may facilitate the recall of extinction in the absence of the mPFC, we have explored the $\mathrm{MO}$, the SEP, and the MD. The MO and the MD have been found to display elevated metabolism in relation to extinction of conditioned fear, indicating that the animals with higher neural activity in these areas were more successful at inhibiting their conditioned freezing response to tone (Barrett et al. 2003). Additionally, high-frequency stimulation of the MD before extinction training has been reported to facilitate retention of extinction, while low-frequency stimulation of the same area during extinction training inhibit the recall of extinction (Herry and Garcia 2002), confirming the function of the MD in inhibitory processes of conditioned fear after extinction. However, despite this evidence (Herry and Garcia 2002; Barrett et al. 2003), our data show that fear extinction can still be expressed without the MO or the MD. Concerning the SEP, several studies that have repeatedly demonstrated that cells in the dorsal septum increase their rates of firing in the presence of stimuli that signal relief or safety and inhibit their rates of firing in the presence of a CS initially paired with footshock (Thomas and Yadin 1980; Yadin and Thomas 1981; Thomas et al. 1991). However, there is no direct evidence of the involvement of this area in fear extinction. Our data, along with data reported by others (Sparks and LeDoux 1995; Barrett et al. 2003), suggest that the SEP is not involved in extinction of freezing to a tone CS. These data also suggest that the mechanisms of conditioned inhibition of fear that activate the SEP (Thomas et al. 1991) are different from the mechanisms involved in extinction of conditioned fear (see also Jones and GonzalezLima 2001; Barrett et al. 2003).

In conclusion, under the conditions used here, lesions of each of the known HPC output targets implicated in fear inhibition (MO, SEP, and MD), combined with mPFC lesions, are not sufficient to disrupt recall of fear extinction $7 \mathrm{~d}$ after training.

\section{Materials and Methods}

\section{Subjects and surgery}

Male Long-Evans rats (Harlan Sprague Dawley), weighing between 250 and $330 \mathrm{~g}$, were housed in individual cages with 14-h light/10-h dark cycle (lights on at 7:00 a.m.), and allowed food and water ad libitum. During the first $5 \mathrm{~d}$, they were handled for $15 \mathrm{sec} / \mathrm{d}$ to habituate them to the experimenter. Rats received either post-extinction or pre-conditioning lesions or were sham operated $(\mathrm{SH})$. In both cases, rats were anesthetized with sodium pentobarbital (Nembutal, $65 \mathrm{mg} / \mathrm{kg}$, i.p.), treated with atropine $(0.04 \mathrm{mg} / \mathrm{kg}$, i.p.), and placed in a stereotaxic frame for electro- 
lytic lesions with stainless-steel electrodes insulated with Epoxylite, except for $0.5 \mathrm{~mm}$ at the tip. Electrodes targeted these regions (coordinates relative to bregma): the $\mathrm{mPFC}(\mathrm{AP},+2.7 \mathrm{~mm}$; $\mathrm{ML}, \pm 0.5 \mathrm{~mm}$; DV,$-5.2 \mathrm{~mm}$ ), the dorsal (AP, $+2.2 \mathrm{~mm}$; ML, $\pm 0.8 \mathrm{~mm} ; \mathrm{DV},-1.8)$ and lateral $(\mathrm{AP},+2.2 \mathrm{~mm} ; \mathrm{ML}, \pm 3.0 \mathrm{~mm}$; $\mathrm{DV},-2.0 \mathrm{~mm}) \mathrm{MO}$, the SEP $(\mathrm{AP},+0.6 ; \mathrm{ML}, \pm 0.4 \mathrm{~mm} ; \mathrm{DV},-5.3$ $\mathrm{mm})$, or the medial part of the MD (AP, $-2.5 \mathrm{~mm}$; ML, \pm 0.8 $\mathrm{mm} ; \mathrm{DV},-5.6 \mathrm{~mm})$. Combined lesions included the mPFC and one of the other areas $(\mathrm{MO}+\mathrm{mPFC}, \mathrm{SEP}+\mathrm{mPFC}$, and $\mathrm{MD}+\mathrm{mPFC})$. Lesions were made with anodal constant direct current at each location (1.2 mA for $12 \mathrm{sec}$ or $1 \mathrm{~mA}$ for $12 \mathrm{sec}$ for SEP lesions), and the incision was closed with stainless-steel wound clips. The rats were allowed to recover for $7 \mathrm{~d}$.

\section{Apparatus}

Eight identical observation chambers $(30 \times 24 \times 21 \mathrm{~cm}$; MEDAssociates) were used in all experiments. The chambers were constructed of aluminum (side walls) and Plexiglas (rear wall, ceiling, and hinged front door) and were situated in sound-attenuating cabinets located in a brightly lighted and isolated room. The floor of each chamber consisted of 19 stainless steel rods (4 $\mathrm{mm}$ in diameter) spaced $1.5 \mathrm{~cm}$ apart (center to center). Rods were wired to a shock source and solid-state grid scrambler (MEDAssociates) for the delivery of footshock US $(0.5 \mathrm{sec}, 1 \mathrm{~mA})$. A speaker mounted outside a grating in one wall of the chamber was used for the delivery of acoustic CS ( $2 \mathrm{sec}, 80 \mathrm{~dB}, 2 \mathrm{kHz})$. Two distinct contexts were used: context $\mathrm{A}$ and $\mathrm{B}$. For context $\mathrm{A}, \mathrm{a}$ $15-\mathrm{W}$ houselight mounted opposite the speaker was turned on, and room lights remained on. The chambers were cleaned with a $1 \%$ acetic acid solution. To provide a distinct odor, stainless steel pans containing a thin layer of this solution were placed underneath the grid floors before the rats were placed inside. Ventilation fans in each chest supplied background noise (65 dB, A scale). Rats were transported to this context in white plastic boxes. For context B, three 40-W red lights were the only source of illumination, the ventilation fans were turned off, the chambers were cleaned with a $1 \%$ ammonium hydroxide solution, and stainless-steel pans containing a thin layer of this solution were placed underneath the grid floors before the rats were placed inside to provide a distinct odor. Rats were transported to this context in black plastic boxes. Additionally, each conditioning chamber rested on a load-cell platform that was used to record chamber displacement in response to each rat's motor activity, therefore allowing detection of freezing behavior via a Threshold-Activity software (MED-Associates). Freezing was quantified (Maren 1998) by computing the number of observations for each rat that had a value less than the freezing threshold (load-cell activity $=5$; animals exhibit freezing when load cell activity is at or below this value). Freezing was determined during the 60 -sec interval after the 2-sec CS presentation during conditioning, extinction, and the retention test, and during the 60 -sec interval preceding the first CS presentation during extinction training.

\section{Procedure}

Rats were submitted to three phases of training: fear conditioning, extinction, and extinction retention test. In each group of rats, $\sim 50 \%$ of animals were conditioned in context $\mathrm{A}$, trained for extinction in context $\mathrm{B}$, and tested for extinction retention in context $\mathrm{B}$, while the remaining subjects were conditioned in context $\mathrm{B}$, trained for extinction in $\mathrm{A}$, and tested for extinction retention in A. For fear conditioning, rats received five tonefootshock trials (62-sec intertrial interval) beginning $3 \mathrm{~min}$ after being placed in the chambers. Rats were returned to their home cages $3 \mathrm{~min}$ after the final shock. The following day, groups receiving extinction training received 45 tone-alone presentations. The no-extinction groups were placed in the chamber for the same amount of time but were not exposed to the tone CS. Seven days following extinction training, all rats were returned to the extinction context and exposed to five CS-alone presentations.

\section{Histology and data analysis}

Histological verification of lesion location was performed after behavioral testing. Rats were perfused across the heart with physiological saline followed by a $10 \%$ formalin solution. After extraction from the skull, brains were post-fixed in 10\% formalin solution for $2 \mathrm{~d}$, at which time the solution was replaced with a $10 \%$ formalin/30\% sucrose solution until sectioning. Sections $\left(50-\mu \mathrm{m}\right.$ thick) were cut on a cryostat $\left(-20^{\circ} \mathrm{C}\right)$ and stained with $0.25 \%$ thionin for visualization of lesion. All behavioral data are expressed as means and standard error of the means (SE) and analyzed by analysis of variance (ANOVA).

\section{Acknowledgments}

This work was supported by the following grants: NIH (R01MH065961) to S.M., Philippe Foundation grant to R.G.

\section{References}

Barrett, D., Shumake, J., Jones, D., and Gonzalez-Lima, F. 2003. Metabolic mapping of mouse brain activity after extinction of a conditioned emotional response. J. Neurosci. 23: 5740-5749.

Garcia, R. 2002. Postextinction of conditioned fear: Between two CS-related memories. Learn. Mem. 9: 361-363.

Gewirtz, J.C., Falls, W.A., and Davis, M. 1997. Normal conditioned inhibition and extinction of freezing and fear-potentiated startle following electrolytic lesions of medical prefrontal cortex in rats. Behav. Neurosci. 111: 712-726.

Herry, C. and Garcia, R. 2002. Prefrontal cortex long-term potentiation, but not long-term depression, is associated with the maintenance of extinction of learned fear in mice. J. Neurosci. 22: 577-583.

Herry, C. and Mons, N. 2004. Resistance to extinction is associated with impaired immediate early gene induction in medial prefrontal cortex and amygdala. Eur. J. Neurosci. 20: 781-790.

Herry, C., Vouimba, R.M., and Garcia, R. 1999. Plasticity in the mediodorsal thalamo-prefrontal cortical transmission in behaving mice. J. Neurophysiol. 82: 2827-2832.

Hugues, S., Deschaux, O., and Garcia, R. 2004. Postextinction infusion of a mitogen-activated protein kinase inhibitor into the medial prefrontal cortex impairs memory of the extinction of conditioned fear. Learn. Mem. 11: 540-543.

Jones, D. and Gonzalez-Lima, F. 2001. Associative effects of Pavlovian differential inhibition of behaviour. Eur. J. Neurosci. 14: 1915-1927.

Lebron, K., Milad, M.R., and Quirk, G.J. 2004. Delayed recall of fear extinction in rats with lesions of ventral medial prefrontal cortex. Learn. Mem. 11: 544-548.

Maren, S. 1998. Overtraining does not mitigate contextual fear conditioning deficits produced by neurotoxic lesions of the basolateral amygdala. J. Neurosci. 18: 3088-3097.

Milad, M.R. and Quirk, G.J. 2002. Neurons in medial prefrontal cortex signal memory for fear extinction. Nature 420: 70-74.

Morgan, M.A. and LeDoux, J.E. 1995. Differential contribution of dorsal and ventral medial prefrontal cortex to the acquisition and extinction of conditioned fear in rats. Behav. Neurosci. 109: 681-688.

Morgan, M.A., Romanski, L.M., and LeDoux, J.E. 1993. Extinction of emotional learning: Contribution of medial prefrontal cortex. Neurosci. Lett. 163: 109-113.

Pavlov, I.P. 1927. Conditioned reflexes. Oxford University Press, Oxford, UK.

Quirk, G.J., Russo, G.K., Barron, J.L., and Lebron, K. 2000. The role of ventromedial prefrontal cortex in the recovery of extinguished fear. J. Neurosci. 20: 6225-6231.

Santini, E., Ge, H., Ren, K., Pena de Ortiz, S., and Quirk, G.J. 2004. Consolidation of fear extinction requires protein synthesis in the medial prefrontal cortex. J. Neurosci. 24: 5704-5710.

Sparks, P.D. and LeDoux, J.E. 1995. Septal lesions potentiate freezing behavior to contextual but not to phasic conditioned stimuli in rats. Behav. Neurosci. 109: 184-188.

Thomas, E. and Yadin, E. 1980. Multiple-unit activity in the septum during Pavlovian aversive conditioning: Evidence for an inhibitory role of the septum. Exp. Neurol. 69: 50-60.

Thomas, E., Yadin, E., and Strickland, C.E. 1991. Septal unit activity during classical conditioning: A regional comparison. Brain Res. 547: $303-308$

Vouimba, R.M., Garcia, R., Baudry, M., and Thompson, R.F. 2000. Potentiation of conditioned freezing following dorsomedial prefrontal cortex lesions does not interfere with fear reduction in mice. Behav. Neurosci. 114: 720-724.

Yadin, E. and Thomas, E. 1981. Septal correlates of conditioned inhibition and excitation in rats. J. Comp. Physiol. Psychol. 95: $331-340$.

Received August 18, 2005; accepted in revised form October 7, 2005. 


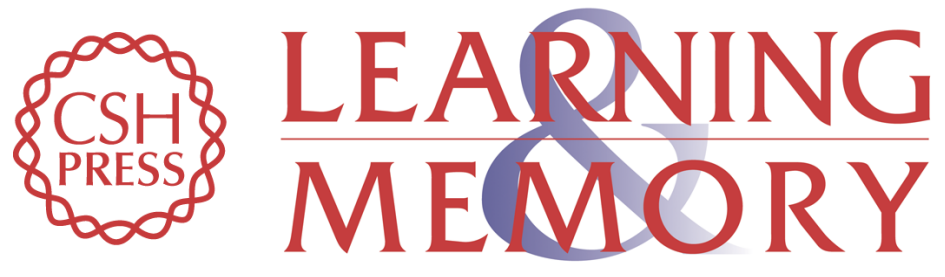

\section{Electrolytic lesions of the medial prefrontal cortex do not interfere with long-term memory of extinction of conditioned fear}

René Garcia, Chun-hui Chang and Stephen Maren

Learn. Mem. 2006, 13:

Access the most recent version at doi:10.1101/lm.60406

References This article cites 20 articles, 8 of which can be accessed free at: http://learnmem.cshlp.org/content/13/1/14.full.htmI\#ref-list-1

License

Email Alerting Receive free email alerts when new articles cite this article - sign up in the box at the Service top right corner of the article or click here. 